Casos Clínicos

Arch. Esp. Urol., 59, 1 (78-81), 2006

\section{MICROLITIASIS TESTICULAR.}

\author{
Nuria Rodríguez García, Inmaculada Fernández \\ González', Carlos Pascual Mateo, Gino Espinales \\ Castro, Javier Angulo Cuesta y Antonio Berenguer \\ Sánchez.
}

Servicio de Urología. Hospital Universitario de Getafe. Getafe y Servicio de Urología'. Hospital de la Princesa. Madrid. España.

Resumen.- OBJETIVO: La microlitiasis testicular (MT), que se caracteriza por la existencia de microcalcificaciones dentro de los túbulos seminíferos, es poco frecuente y generalmente constituye un hallazgo incidental durante la realización de la ecografía escrotal.

MÉTODO: Presentamos un caso de un varón de 45 años de edad, sin factores de riesgo para el desarrollo de un tumor testicular de células germinales, diagnosticado de una microlitiasis testicular bilateral.

RESULTADOS: El paciente ha sido sometido a control evolutivo anual con ecografía testicular, sin desarrollar un tumor germinal durante un seguimiento de 4 años.

CONCLUSIONES: Aunque el significado clínico de la MT está en debate debido a las diferentes controversias halladas en la literatura sobre su asociación o no con el tumor testicular, así como los diferentes protocolos de manejo inicial y de los intervalos adecuados para su vigilancia y seguimiento, parece razonable realizar un control ecográfico anual, independientemente de la existencia o no de factores de riesgo asociados.
Palabras clave: Microlitiasis testicular

Summary.- OBJECTIVES: Testicular microlithiasis, characterized by the existence of microscopic calcifications within the seminiferous tubules, is rare, generally an incidental finding during a scrotal ultrasound.

METHODS: We report the case of a 45-year-old male without risk factors for the development of a germ cell testicular tumor with the diagnosis of bilateral testicular microlithiasis.

RESULTS: The patient was followed yearly with testicular ultrasound, without evidence of germ cell tumor over a fouryear follow-up period.

CONCLUSIONS: Although the clinical significance of testicular microlithiasis is under debate due to the various controversies found in the literature about its association or not with testicular tumors, as well as the various protocols for initial management and adequate intervals for followup, it seems reasonable to perform an ultrasound yearly independently of the existence or not of associated risk factors.

Keywords: Testicular microlithiasis.

\section{INTRODUCCIÓN}

La microlitiasis testicular (MT), que se caracteriza por la existencia de microcalcificaciones dentro de los túbulos seminíferos, es poco frecuente y generalmente constituye un hallazgo incidental durante la realización de la ecografía escrotal. El significado clínico de la MT está en debate debido a las diferentes controversias halladas en la literatura sobre su asociación con el tumor testicular, así como los diferentes protocolos de tratamiento inicial y los intervalos adecuados para su vigilancia y seguimiento $(1,5)$.

\section{CASO CLÍNICO}

Varón de 45 años de edad, sin antecedentes personales de interés que acude a la consulta de urología por dolor testicular bilateral de 4 años de evolución. La exploración física es normal y la ecografía escrotal realizada con un transductor de alta frecuencia (10 Mhz) pone de manifiesto la presencia de una MT bilateral (Figura 1). Debido a la no existencia de factores de riesgo (criptorquidia, atrofia, infertilidad, neoplasia intratubular de células germinales, disgenesia gonadal, tumor testicular contralateral, administración exógena de estrógenos) se decide seguimiento con autoexploración física mensual y valoración urológica con ecografía escrotal anual, sin realización de biopsia. A los 4 años de seguimiento no ha desarrollado un tumor invasivo de células germinales. 


\section{DISCUSIÓN}

La MT, que se caracteriza por la existencia de microcalcificaciones dentro de los túbulos seminíferos, es poco frecuente y generalmente es un hallazgo incidental durante la realización de la ecografía escrotal. El análisis ultraestructural pone de manifiesto la presencia de un núcleo central calcificado rodeado por capas concéntricas de tejido conectivo o fibras de colágeno; estos hallazgos sugieren que los microlitos se originan por acumulación intratubular de restos celulares, seguida de la disposición de glicoproteínas alrededor del núcleo y posterior calcificación de este material (6).

La definición generalmente aceptada de la MT es la presencia de 5 o más pequeños focos ecogénicos dentro del testículo con un tamaño que oscila entre 1 y $3 \mathrm{~mm}$ en diámetro (5). No afectan a la forma ni al tamaño del testículo. Carecen generalmente de sombra acústica, lo que se debe probablemente a su pequeño tamaño; este hecho es una característica diagnóstica importante para distinguirlos de otras calcificaciones intratesticulares. Existe un sistema de clasificación de la MT basado en el número de microlitos existentes por testículo: Grado I (5-10 microlitos), Grado 2 (10-20 microlitos) y Grado 3 ( > 20 microlitos) $(5,7)$.

Es importante hacer el diagnóstico diferencial con las patologías que pueden originar calcificaciones intratesticulares. Dentro de las calcificaciones "benignas" se encuentran las originadas por los granulomas post-inflamatorios, las calcificaciones vasculares, los flebolitos, los hematomas, la tuberculosis, la sarcoidosis y los infartos crónicos. El infarto crónico testicular se caracteriza por la presencia de un "grupo" de calcificaciones asociados a un área hipoecoica de localización periférica y con forma de cuña de bordes bien definidos, que denotan la presencia de áreas de necrosis. Las calcificaciones "malignas" se asocian al tumor de células germinales "burnerd-out", al teratoma, al teratocarcinoma, al tumor de células de Sertoli y menos frecuentemente al seminoma (8).

La prevalencia de la MT en la población asintomática basada en los hallazgos ecográficos varía entre el 0,6\% al $5,6 \% 9,(10)$. Valores similares se han recogido en la población subfértil: $0,8 \%-9,5 \%(11-14)$ y en la población que acude al urólogo 0,7 - 4,1\% $(15,16)$.

La mayoría de los autores no creen que la MT por sí misma sea una lesión premaligna, pero sí representa una anormalidad testicular que pueda predisponer a la malignidad. Backus y cols. (5) observaron que el $29 \%$ de los pacientes con MT desarrolla tumores testiculares, siendo los más frecuentes los seminomas, los teratomas y tumores mixtos de células germinales (10). Hobarth y cols. (2) observaron que el $45 \%$ de los pacientes con MT tenían un tumor testicular. Otite y cols. (17) afirman que el riesgo relativo de tener un tumor testicular, cuando existe una MT, es de 13,2. Raramente, sin embargo, la MT es diagnosticada antes del desarrollo de un cáncer testicular, siendo el tiempo medio hasta que aparece un tumor testicular clínicamente aparente de 48 meses (rango 10-132) (18-22). Holm y cols. (23) detectaron un riesgo incrementado de carcinoma in situ (CIS) en el testículo contralateral cuando existía MT en pacientes con tumor de células germinales (odds ratio 28.6; Cl: 4.8-170.4). De Gouveia Brazao y cols. (24) indican seguimiento con ecografía o biopsia en pacientes subfértiles en los que la MT es bilateral, por el riesgo

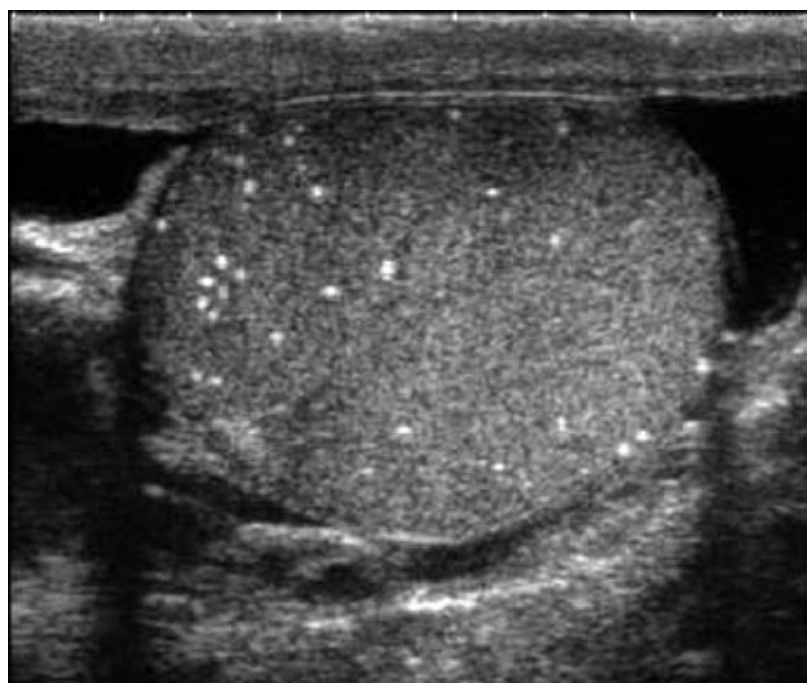

FIGURA 1a. Ecografía escrotal con transductor de alta frecuencia (10 Mhz) y en escala de grises donde se observa la microlitiasis testicular.

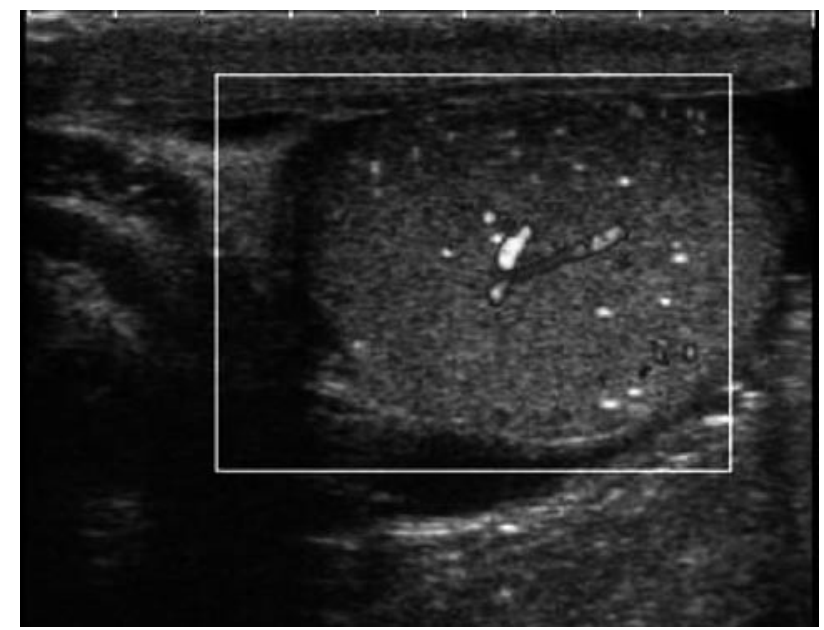

FIGURA 1b. Ecografía testicular con Eco Doppler color; las calcificaciones no se corresponden con calcificaciones vasculares 
de desarrollar un tumor testicular invasivo. Sin embargo Peterson y cols. (9) y Furness y cols. (10) no encuentran asociación entre MT y tumor testicular en aquellos pacientes asintomáticos.

El significado clínico de la MT está en debate debido a las diferentes controversias halladas en la literatura y también por su manejo. Dicha controversia implica la exitencia de distintas conductas, desde no realizar ningún tipo de seguimiento hasta practicar TAC torácica y abdomino-pélvica y biopsia del testículo afectado $(9$, $21,25,26)$. La monitorización de los marcadores séricos tumorales en ausencia de otras indicaciones no es adecuada debido a que la mayoría de los estudios no han encontrado valores elevados en aquellos casos de diagnóstico incidental de MT (9).

Rashid y cols. (27) han propuesto un protocolo para el seguimiento y control en pacientes que presentan una MT. Aquellos con factores de riesgo conocidos para desarrollar un tumor testicular (criptorquidia, atrofia, infertilidad, neoplasia intratubular de células germinales, disgenesia gonadal, la existencia de un tumor testicular contralateral y la administración exógena de estrógenos), son sometidos a autoexploración física mensual y revisión anual por un urólogo con realización de ecografía escrotal; en aquellos casos sin factores de riesgo, autoexploración física y valoración anual por el médico de familia, remitiéndolo al urólogo en caso de observar alguna anomalía en la evolución.

\section{CONCLUSIONES}

Aunque el significado clínico de la MT está en debate debido a las diferentes controversias encontradas en la literatura sobre su asociación o no con el tumor testicular, así como los diferentes protocolos de manejo inicial y de los intervalos adecuados para su vigilancia y seguimiento, parece razonable realizar un control ecográfico anual, independientemente de la existencia o no de factores de riesgo asociados.

\section{BIBLIOGRAFÍA Y LECTURAS RECOMENDADAS (*lectura de interés $y^{* *}$ lectura fundamental)}

1. IKINGER, U.; WURSTER, K.; TERWEY, B.: "Microcalcifications in testicular malignancy". Urology, 19: $525,1982$.

2. HOBARTH, K.; SUSANI, M.; SZABO, N. y cols.: "Incidence of testicular micro-lithiasis". Urology, 40: 464, 1992.

3. BACH, A.M.; HANN, L.E.; HADAR, O. y cols.: "Testicular microlithiasis: what is its association with testicular cancer?". Radiology, 220: 70, 2001.

4. RENSHAW, A.A.: "Testicular calcifications". J. Urol., 160: 1625, 1998.
5. BACKUS, M.L.; MACK, L.A.; MIDDLETON, W.D. y cols.: "Testicular microlithiasis: imaging appearances and pathologic correlation". Radiology, 192: 781, 1994.

6. NISTAL, M.; PANIAGUA, R.; DÍEZ-PARDO, J.A.: "Testicular microlithiasis in 2 children with bilateral cryptorchidism". J. Urol., 121: 535, 1979.

7. CORNUD, F.; AMAR, E.; HAMIDA, K. y cols.: "Ultrasound findings in male hypofertility and impotence". Eur. Radiology, 11: 2126, 2001.

8. MILLER, F.N.; SIDHU, P.S.: "Does testicular microlithiasis matter? A review". Clin. Radiol., 57: 883, 2002.

9. PETERSON, A.C.; BAUMAN, J.M.; LIGHT, D.E. y cols.: "The prevalence of testicular microlithiasis in an asymptomatic population of men 18 to 35 years old". J. Urol., 166: 2061, 2001.

10. FURNESS, P.D.III.; HUSMANN, D.A.; BROCK, J.W. III. y cols.: "Multi-institutional study of testicular microlithiasis in childhood: a benign or premalignant condition?". J. Urol., 160: 1151, 1998.

11. NICOLAS, F.; DUBOIS, R.; LABOURE, S. y cols.: "Testicular microlithiasis and chryptorchidism: ultrasound analysis after orchidopexy". Prog. Urol., 11: 357, 2001.

12. SCHREY, A.; REITER, W.J.; KRATZIK, C.: "Value of ultrasound in microlithiasis of the testis in andrological patients". Ultraschall. Med., 22: 143, 2001.

13. THOMAS, K.; WOOD, S.J.; THOMPSON, A.J. y cols.: "The incidence and significance of testicular microlithiasis in a subfertile population". Br. J. Radiol., 73: 494, 2000.

14. PIERIK, F.H.; DOHLE, G.R.; VAN MUISWINKEL, J.M. y cols.: "Is routine scrotal ultrasound advantageous in infertile men?". J. Urol., 162: 1618, 1999.

15. DEROGEE, M.; BEVERS, R.F.; PRINS, H.J. y cols.: "Testicular microlithiasis, a premalignant condition: prevalence, histopathologic findings, and relation to testicular tumor". Urology. 57: 1133, 2001.

16. GANEM, J.P.; WORKMAN, K.R.; SHABAN, S.F.: "Testicular microlithiasis is associated with testicular pathology". Urology, 53: 209, 1999.

17. OTITE, U.; WEBB, J.A.; OLIVER, R.T. y cols.: "Testicular microlithiasis: is it a benign condition with malignant potential?". Eur. Urol., 40: 538, 2001.

18. WINTER, T.C. III.; ZUNKEL, D.E.; MACK, L.A.: "Testicular carcinoma in a patient with previously demonstrated testicu-lar microlithiasis". J. Urol., 155: 648, 1996.

19. SALISZ, J.A.; GOLDMAN, K.A.: "Testicular calcifications and neoplasia in patient treated for subfertility". Urology, 36: 557, 1990.

20. GOODING, G.A.W.: "Detection of testicular microlithiasis by sonography". AJR Am. J. Roentgenol., 168: 281, 1997.

21 FRUSH, D.P.; KLIEWER, M.A.; MADDEN, J.F.: "Testicular mi-crolithiasis and subsequent development of metastatic germ cell tumor". AJR Am. J. Roentgenol., 167: 889, 1996. 
22. McENIFF, N.; DOHERTY, F.; KATZ, J. y cols.: "Yolk sac tumor of the testis discovered on a routine annual sonogram in a boy with testicular microlithiasis". AJR Am. J. Roentgenol., 164: 971, 1995.

23. HOLM, M.; HOEI-HANSEN, C.E.; RAJPERT-DE MEYTS, E. y cols.: "Increased risk of carcinoma in situ in patients with testicular germ cell cancer with ultrasonic microlithiasis in the contralateral testicle". J. Urol., 170: 1163, 2003.

24. DE GOUVEIA BRAZAO, C.A.; PIERIK, F.H.; OOSTERHUIS, J.W. y cols.: "Bilateral testicular microlithiasis predicts the presence of the precursor of testicular germ cell tumors in subfertile men". J. Urol., 171: 158, 2004.

25. WITTENBERG, K.H.; HATTERY, R.R.; GORMAN, B. y cols.: "Testicular microlithiasis and testicular tumours: association or pre-malignant condition". Radiology, 217: 366, 2000.

26. WEGNER, H.E.; HAMM, B.; LOY, V. y cols.: "Testicular micro-lithiasis: case report and discussion of management under special consideration of germ cell tumours". Int. Urol. Nephrol., 28: 533, 1996.

27. RASHID, H.H.; COS, L.R.; WEINBERG, E. y cols.: "Testicular microlithiasis: a review and its association with testicular cancer". Urol. Oncol., 22: 285, 2004.
Casos Clínicos

Arch. Esp. Urol., 59, 1 (81-84), 2006

\section{LEIOMIOMA RENAL: APORTACIÓN DE UN NUEVO CASO.}

Angel Andreu García, Enrique Herrero Polo, José Miguel Alapont Alacreu, Mํa Belén Coronel Sánchez, Enrique Schiefenbuch Munné, Roberto Botella Almodovar, José Luis Brotons Márquez y Gonzalo Llamazares Cachá.

Servicio de Urología. Hospital Virgen de la Salud de Elda. Elda. Alicante. España.

Resumen.- OBJETIVO: Aportar un nuevo caso de leiomioma capsular asintomático.

MÉTODO: Paciente de 68 años asintomático que tras estudio radiológico (ECO y TAC) es diagnosticada de masa sólida.

RESULTADO: El tratamiento de este proceso neoformativo renal, fue nefrectomía radical, con curso postoperatorio sin incidencias y anatomía patológica, leiomioma renal.

CONCLUSIÓN: El leiomioma renal es un fumor mesenquimatoso benigno poco frecuente que debe tenerse en cuenta ante el diagnóstico de masa renal, dado que sería subsidiario de una cirugía renal conservadora, no obstante ante la dificultad para diferenciarlo del carcinoma renal, no es infrecuente que el diagnóstico se produzca tras el análisis histológico de la pieza de nefrectomía.

Palabras clave: Leiomioma. Cápsula renal. Tumores renales benignos. 